\title{
Taking Excimer Laser Processing to Industry Perspectives from a NATO Advanced Study Institute
}

\author{
Lucien D. Laude
}

A NATO Advanced Study Institute entirely devoted to excimer lasers took place September 7-16, 1993 in Elounda (Crete), Greece, gathering some 85 scientists from 21 different countries, mostly from Germany, France, and Greece. Among the continuously expanding list of laser sources, excimer lasers are unique in their technical specifications, venturing into unusual fundamental physics and chemistry, and thus opening routes to novel applications. Emitting intense pulses of uv light, excimer lasers are best characterized by their enormous instant power (tens of megawatts) delivered in a dense flux of high-energy photons able to overcome bond strength. This gives access to processes like nonequilibrium (thermal or athermal) surface phase transitions or chemical reactions which are not accessible either to other laser sources or via conventional (thermodynamic) processes. In addition, and most remarkably, these excimer sources are equally able to process (i.e., to transform irrevocably) materials as diverse as ceramics, polymers, metals, or biological tissues. As a result, a single excimer laser beam may find equally effective applications in fields as diverse as materials science and medicine. For example, one of the many processes that can be induced by excimerlaser irradiating matter, namely ablation, has proved to be a reliable and simple way to produce good-quality high Tc superconducting films, custom-designed 3-D engraving on either hard or soft materials, and high-precision ceramic machining. In medicine, novel techniques like corneal reshaping, cancer phototherapy, and others can be likewise elaborated. The extreme versatility of the excimer laser is in itself unique in the field of lasers, and a fortiori in the broader panoply of energy sources which have been developed and utilized until now.

However, full development of excimer laser potential may only be achieved under three conditions: (1) the characteristics and limitations of the sources should be known to all practitioners; (2) a reasonable understanding of the basic processes which are induced by excimerirradiating matter should be disseminated throughout the larger community; and (3) technological or industrial problems in search of a solution should be clearly identified.

Scientists were not, are not, and will never be able to transfer their occasional bright ideas efficiently to the industrialists, who logically should be their customers, unless they direct some serious effort toward the real world.

The first two conditions are within reach of scientists as part of their educational background. However, because of the extreme diversity or complexity of the disciplines involved, the conditions for the dissemination of knowledge (either technical or scientific) are not currently fulfilled through the usual specialty conference series, which do not allow for cross-fertilization.

One objective of this NATO Advanced

Material Matters is a forum for expressing personal points of view on issues of interest to the materials community
Study Institute was to promote an interdisciplinary exchange of information among all scientists, R\&D researchers, fundamentalists, technologists, life science practioners, and environmentalists on the physics and chemistry of excimer laser interactions with matter. The program covered the following topics:

1. The state of the art in excimer laser technology;

2. Fundamental aspects of the interaction of such beams with matter, particularly ablation;

3. Experimental diagnostics used routinely in the field;

4. Applications of excimer irradiation in materials science, spanning microelectronics, metallurgy, ceramics, and polymers;

5. Applications in the medical field; and 6. Further applications in environmental science.

Another objective of the Institute was to address the third condition-identification of valuable problems to investigate in the context of transfer from science to technology and, further, to industry. The theme was extensively discussed during the course of the two-week meeting, particularly in presentations by $\mathrm{F}$. Bachmann (Rofin-Sinar Laser $\mathrm{GmbH}$, Hamburg) and a few others. The excitement experienced during these discussions was indicative of an overwhelming concern among the participants. And several fruitful ideas were aired, sometimes coming from the younger participants. Which problem to address? Where to place the priorities?

Identifying valuable problems is probably the most difficult task for the majority of those scientists, though any successful transfer of technology would directly depend on a proper choice of such problems "in search of a solution." Lasers are rightly known to provide a number of elegant ways to act on matter in whichever form. However, all these reported "laser-induced processes" must, at some point, compete with existing technology in order to find their specific applications. As surprising as it is, most R\&D laser practitioners have not (yet) fully realized that aspect of their work. In general, technologists didn't wait for the advent of lasers as energy sources to solve technological problems at the production level. Given the investments already made in solving such problems, say, classically and sometimes imperfectly (but sufficiently), laser processes must prove to be not simply as good as competing (but already established) processes. They must be either (1) much more 
effective (an order of magnitude at least), in terms of product quality, production cost, friendliness at the bench, and reliability at the production level; or (2) absolutely unavoidable for producing a brand new device. Unfortunately, such novelty emerges from laboratories where a market attitude is totally absent. Scientists were not, are not, and will never be able to transfer their occasional bright ideas efficiently to the industrialists, who logically should be their customers, unless they direct some serious effort toward the real world.

Scientists are, in general, unaware of the true needs and trends of the market. Still, the gap must be closed and every actor ("inventor" or "producer") in this "technology transfer drama" should try and try again, first, to understand the other side and, second, to come to some compromise on the issue. Lasers, and most readily excimer lasers, may effectively provide for true revolutionary steps toward progress in a variety of domains. However, advancement in economy may be anything but revolutionary. Economy is fragile and works under near-equilibrium conditions. The reported difficulties in implementing laser processes in technology stem from both attitudes: (1) the apparent unawareness by the "inventors" of this legitimate and natural fact, and (2) the reluctance of the "producers" to disturb the fragile equilibrium of a production-line because it often takes time and investment to tune up to a given market product. These two attitudes are common to all fields, from metallurgy to ophthalmology. Should the necessity of competition between producers on one hand and the emergence of new and more demanding specifications on the other hand require novel products or processes, then the excimer laser may prove to be the effective tool to be implemented.

Since the opportunity might show up at any time, one should be ready at any moment to propose, test, and transfer a process which could earlier have been "invented," understood, and developed for that expectation-but stems instead from fundamental studies not motivated toward that goal. Even so, the proposed process should follow elemental, common sense rules: conceptual simplicity (two-step processing at a maximum), low cost, easy implementation, compatibility with existing technology, and last but not least, avoidance of solutions if there is no need for one.

The matter would be common to many domains of science, as soon as $R \& D$ developments emerge which are thought to provide for new possibilities of application. It is probably more critically felt in the laser field because of the widespread publicity given to this energy source. However, it was the general opinion at the end of the Institute that many more applications would succeed in finding a niche if the rules in the previous paragraph were followed. For that reason, one may hope that this Institute has been of some help to the young participants in maturing the field of excimer laser processing for a more fruitful harvest in the years to come.

This overview of the meeting would not be complete without deeply acknowledging the help provided by the NATO Scientific Affairs Division to its venue. Substantial and decisive, that help was primarily financial. Which other organization in the world could provide today for that generous support to science? But the help was not simply finan- cial. Meetings of this type are essential to higher education. Teaching wonders of the past does not necessarily prepare for the future. Educating means opening the mind, and for that we need to step away from our everyday burden and take time to think again. Listen to the questions of the younger scientists, provide them with keys to the answers, and raise among them the curiosity for the facts which are mother to innovation. And somewhere hidden in the audience, away from the noise and turmoil, ideas will emerge, paving the way toward wonders of the future. These will ultimately be the most rewarding fruits of that meeting.

L.D. Laude is a professor in the Laboratory of Solid State Physics at the University of Mons in Belgium and was director of the NATO Advanced Study Institute on Excimer Lasers.

\section{BAIZERS KELCOOL ${ }^{\mathrm{TM}} 4.2 \mathrm{GM} .$. THE ONLY WAY TO 4.2K}

The easy way to optimize your cooling applications with the only standard two-stage, Gifford-McMahon cryocooler that delivers $0.5 \mathrm{~W}$ working capacity at $4.2 \mathrm{~K}$. A simple design provides high refrigeration capacity without the high cost and complexity of a third stage. Ideal for cooling R\&D samples, MRI, superconducting magnet shields, IR detectors, cryopumping and gas liquefaction.

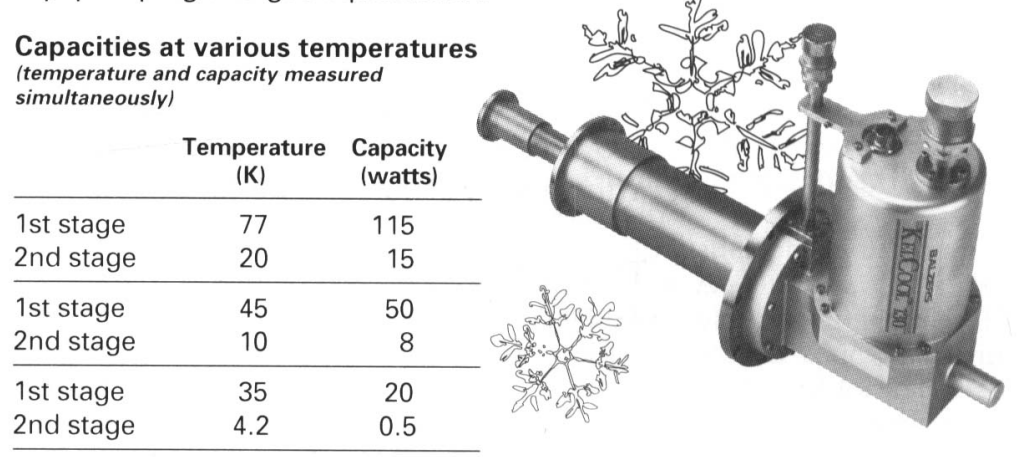

\section{Call us today for all the cold facts!}

Balzers

8 Sagamore Park Road

Hudson, NH 0305

TEL (603) 889-6888

FAX (603) 889-8573

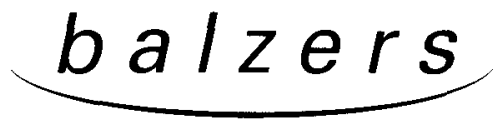

Circle No. 11 on Reader Service Card. 


\section{FULL SPECTRUM FIDELITY}

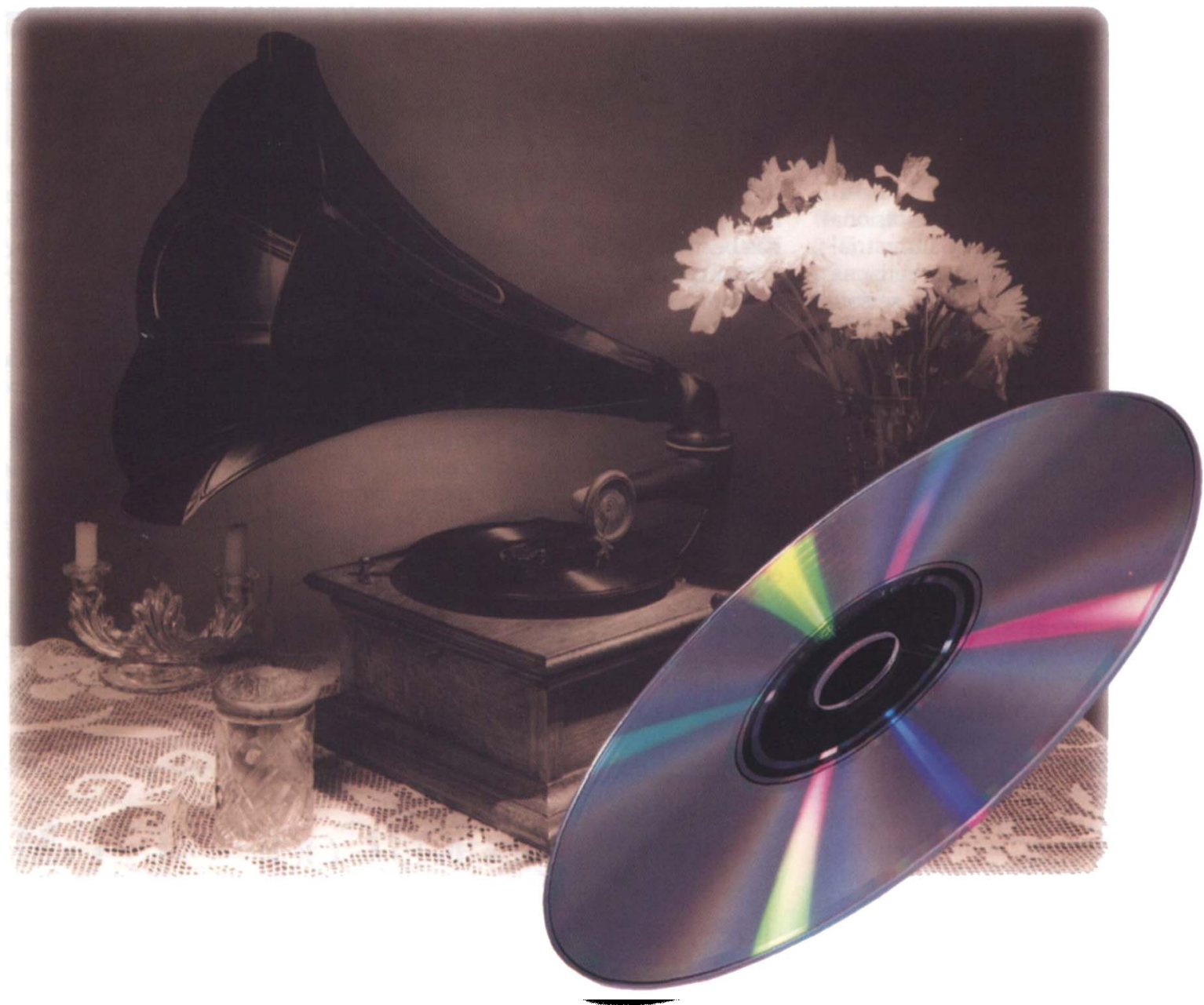

\section{PRISM $^{\mathrm{TM}}$ Digital Pulse Processing for EDS}

What the CD did for music, the PRISM

Digital Spectrometer will do for X-ray analysis.

Noise-free digital signal processing provides extraordinary enhancement in the quality of detector performance.

This landmark development in $\mathrm{X}$-ray pulse processing gives substantial improvements in sensitivity, precision and total detection efficiency for all elements while increasing the productivity of the microanalysis laboratory.

In the future, all EDS systems will use Digital Pulse Processing ...

PGT brings it to you TODAY!

For Information on the PRISM Digital Detector and the New IMIX-XD ${ }^{\mathrm{TM}}$ Microanalysis System contact Princeton Gamma-Tech

\section{PGT Leader from the beginning...Committed to stay in front!}

Princeton Gamma-Tech, Inc. 1200 State Road

Princeton, NJ 08540

TEL: (609) 924-7310

FAX: (609) 924-1729

TELEX: 834486PGTUSA
PGT UK, Ltd.

2, the Metro Centre

Welbeck Way, Woodston

Peterborough, PE2 7UH

TEL: 733 391-811

FAX: 733 391-020

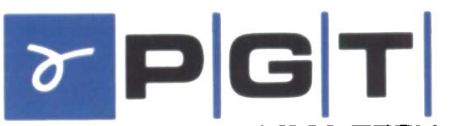

PRINCETON GAMMA-TECH 